\title{
向蟾蜍脊髓灰质腹角注射谷氨酸钠对腓肠肌收缩的影响
}

\author{
陆长亮，张晓萌，祝建平，艾洪滨 ${ }^{*}$ \\ (山东师范大学 生命科学学院, 山东省动物抗性生物学重点实验室, 山东 济南 250014)
}

\begin{abstract}
摘要: 首先绘制了蟾蜍脊髓灰质腹角运动神经元池的精确定位图谱, 然后分别将浓度为 $1 、 0.5 、 0.1 、 0.01 \mathrm{~mol} / \mathrm{L}$ 的谷氨酸钠溶液及生理盐水 $(0.65 \% \mathrm{NaCl}$, 对照组) 微量 $(0.1 \mu \mathrm{L})$ 注射到蛻蜍脊髓灰质腹角运动神经元池, 采 用 BL-420F 生物机能实验系统记录腓肠肌收缩曲线, 以收缩波的上升相持续时间、最大张力、张力变化速率、 下降相持续时间为指标对各组腓肠肌的收缩曲线进行比较。结果：4 组实验组腓肠肌的收缩形式是不同程度的强 直收缩; 各组收缩波的上升相持续时间、最大张力、张力变化速率在一定程度上存在谷氨酸钠量效依赖关系, 且 $1 \mathrm{~mol} / \mathrm{L}$ 组腓肠肌强直收缩的张力、张力变化速率显著大于其他各组, 这可能是由于谷氨酸钠与运动神经元上谷氨 酸受体结合的数量不同造成的。
\end{abstract}

关键词: 蟾蜍脊髓; 灰质腹角; 运动神经元池; L-谷氨酸钠; 腓肠肌; 收缩性

中图分类号: Q426; Q959.53 文献标识码: A 文章编号: 0254-5853-(2010)01-0094-05

\section{Effects of L-Glutamate Microinjection into the Ventral Horn of Spinal Cord Gray Matter on Gastrocnemius Contractility in Toad}

\author{
LU Chang-Liang, ZHANG Xiao-Meng, ZHU Jian-Ping, AI Hong-Bin* \\ (Key Laboratory of Animal Resistance of Shandong Province, College of Life Sciences, Shandong Normal University, Jinan 250014, China)
}

\begin{abstract}
A diagram of motor neuron pool of ventral horn of spinal cord gray matter in toad was first delineated. Different concentrations $(1,0.5,0.1,0.01 \mathrm{~mol} / \mathrm{L})$ of excitatory amino acid L-Glu or physiological saline $(0.65 \% \mathrm{NaCl})$ were then microinjected into the motor neuron pool in a urethane-anaesthetized toad. The contraction curve of the gastrocnemius was then recorded by the BL-420 Physiological Signal Recording. We took the maximal tension, the duration of rising phase, the velocity of tension variation, and the duration of descending phase as the parameters to study the characteristic of gastrocnemius contractility. It was found that the gastrocnemius contractility of all the 4 groups was tetanus but differed in degree, especially the maximal tension, and velocity of tension variation. In contrast to physiological saline, gastrocnemius contracted by the stimulation of L-Glu, and the contraction parameter showed dose-effect relationships except for the duration of descending phase, which was caused by the combination rate of L-Glu and the receptor.
\end{abstract}

Key words:Toad spinal cord; Ventral horn of gray matter; Motor neuron pool; L-Glu; Gastrocnemius; Contractility

大量的研究表明, 在哺乳类动物中, L-谷氨酸 （L-Glu）是中枢内许多部位的神经元使用的一种 兴奋性神经递质。L-谷氨酸几乎可使脑内任何部位 的神经元产生兴奋性反应(Wang et al, 1999), 也是昆 虫及甲壳纲动物神经肌肉接头处的兴奋性神经递 质(Usherwood \& Machili, 1969)。在两栖动物中, 支 配躯干和四肢骨骼肌的运动神经元的胞体和树突
位于脊髓灰质腹角，这些运动神经元是中枢神经系 统调控骨骼肌活动的最后公路, 那么在蟾蜍脊髓灰 质腹角内, 支配腓肠肌的运动神经元胞体膜或树突 膜上是否也存在 $\mathrm{L}-$ 谷氨酸受体, 即它们是否是谷氨 酸敏感神经元。对这些问题的研究, 尚未见文献报 道。目前研究神经元胞体的受体类型主要采用免疫 组化 (Wang et al, 2009) 和微量注射神经递质

收稿日期: 2009-08-11; 接受日期: 2009-11-16

基金项目: 国家自然科学基金资助项目（30770277）

“通讯作者 (Corresponding author), E-mail: physiology@sdnu.edu.cn; Tel: 0531-86185359

第一作者简介: 陆长亮 (1984-), 男, 博士研究生, 研究方向为动物生理学 
(Spencer et al, 1986; Wang et al, 2008) 两种方法。 本研究以蟾蜍腓肠肌的收缩为指标, 向脊髓腹角微 量注射 $\mathrm{L}$ - 谷氨酸钠, 就这一问题进行初步探索, 为 解决两栖类动物脊髓灰质腹角运动神经元胞体膜 或树突膜突触传递的神经机制提供实验依据。

\section{1 材料和方法}

\section{1 实验材料}

1.1.1 实验动物 中华大蟾蜍(Bufo bufogargarizans)产于济南。人工养殖, 体重 85 95 g, 雌雄不 限。

1.1.2 器材和药品 用 $0.65 \%$ 的氯化钠溶液 (两栖 类动物的生理盐水）分别配置浓度为 $1 、 0.5 、 0.1$ 、 $0.01 \mathrm{~mol} / \mathrm{L}$ 的谷氨酸钠 (北京经科化学试剂公司) 溶液, 任氏液、 $\mathrm{BL}-420 \mathrm{~F}$ 生物机能实验系统 (四川 泰盟科技有限公司), 肌肉张力换能器, 常用蛙类 动物手术器械, Stoelting51600 立体定位仪 (Stoelting 公司，美国）。

\section{2 方 法}

1.2.1 蟾蜍脊髓灰质腹角运动神经元池（motor neuron pool）的定位 蟾蜍腹腔注射氨基甲酸乙酯 $(2000 \mathrm{mg} / \mathrm{kg}$ ) 麻醉。打开胸腹腔, 暴露心脏, 心 室内灌流固定。先灌流 50 80 mL生理盐水替换其血 液, 再灌流 $4 \%$ 的甲醛溶液 $100 \mathrm{~mL}$ 。取出冷髓腰骮 膨大节段, 主要是发出坐骨神经的第八九脊髓节段 (Cai et al, 2007), 放入 4\%的甲醛溶液中浸泡 $3 \mathrm{~d}$, 然后在 $-15^{\circ} \mathrm{C}$ 下恒温冷冻切片, 片厚 $40 \mu \mathrm{m}$, 隔一 片取一片, 贴片, 中性红染色, 中性树胶封片。最 后使用MIE显微图像处理软件测定脊髓灰质前角的 精确定位坐标。测量从背正中沟到腹角大型运动神 经元池上边界 (即神经元池的背部边界) 的距离 $\mathrm{H} 1$ 、 下边界 (即神经元池的腹部边界) 的距离 $\mathrm{H} 2$ 、中央 管到大型运动神经元池内侧的距离 L1、外侧距离 $\mathrm{L} 2$, 以及脊髓的背腹径 $\mathrm{H}$ 与左右径 $\mathrm{L}$ (图 1)。同样 方法共处理 6 只蟾蜍, 各组数据用平均数土标准差

$(\bar{x} \pm S)$ 表示, 结果见表 1 。

1.2.2 向腹角运动神经元池内微量注射 L-Glu 和记 录腓肠肌的收缩曲线 取蟾蜍麻醉, 毁脑 (用毁髓 针从枕骨大孔插入, 伸向颅腔内搅动以破坏脑组 织, 但不毁脊髓) 然后俯位腹位固定于蛙板上, 沿 背部正中线剪开皮肤, 暴露脊柱然后暴露脊髓 $(\mathrm{Ai}$, 2004), 撕去软脊膜。分离腓肠肌, 在远侧端肌腱上
结扎一细棉线, 剪断肌腱, 使其离开胫腓骨, 成为 游离端。将蟾蜍放置于立体定位仪下, 将结扎腓肠 肌的棉线连于张力换能器。启用 BL-420F 生物机能 实验系统，参数设置为：增益 $50 \mathrm{mV}$, 时间常数 DC, 高频率波 $20 \mathrm{~Hz}$, 采样率 $100 \mathrm{~Hz}$, 扫描速度 $1.0 \mathrm{~s} / \mathrm{div}$ 。将已拉制好的尖端直径 $50 \mu \mathrm{m}$ 的微细玻 璃管内灌注 $2 \%$ 滂胺天蓝溶液后再吸入 $0.1 \mu \mathrm{L}$ 谷氨 酸钠溶液, 然后固定到立体定位仪上, 并通过硬质 聚乙烯管与微量进样器连接, 使整个系统密闭好, 确保溶液能顺利从尖端流出。找到第八对脊神经背 根, 将已经固定好的微细玻璃管尖端接触到脊髓背 正中沟，记录此时立体定位仪的三维坐标即为“原 点”坐标。稍上提后, 按照 1.2.1 (表 1) 的坐标下 针, 稳定 $10 \mathrm{~min}$ 后注射 $0.1 \mu \mathrm{L}$ 谷氨酸钠, 记录腓 肠肌收缩曲线。待腓肠肌收缩结束后注射浐胺天蓝 留下蓝点, 心脏灌流, 取脊髓, 固定, 切片 (具体 步骤同 1.2.1), 镜检蓝点以确定微细玻璃管尖端是 否在腹角运动神经元池处。结果只统计定位准确的 数据。依据注射谷氨酸钠浓度的不同将蟾蜍分成 5 组, 分别为生理盐水对照组, 以及 $1 、 0.5 、 0.1 、 0.01$ $\mathrm{mol} / \mathrm{L} 4$ 个浓度的谷氨酸钠溶液组, 每组 8 只蟾蜍。 1.2.3 数据统计分析 以强直收缩波的上升相持 续时间、最大张力、张力变化速率、下降相持续时 间为指标对各组腓肠肌的收缩曲线进行比较。从注 射谷氨酸钠后腓肠肌开始收缩到收缩张力达到最 大值为上升相持续时间，从基线到收缩最大值为最 大张力, 张力变化速率 $=$ 最大张力/上升相持续时间。 从最高点到曲线恢复到最低的值且保持稳定不变 为下降相持续时间 (Zeng et al, 2007)。各组所有数

据均采用平均数土标准差 $(\bar{x} \pm S)$ 表示。采用 SPSS13.0 (SPSS Inc. Chicago, Ill., USA) 统计软件进行统计 分析, 各组之间进行 $t$ 检验, $P \leqslant 0.05$ 为差异显著, $P$ $\leqslant 0.01$ 为差异极显著。

\section{2 结 果}

\section{1 脊髓灰质腹角运动神经元池的定位坐标}

我们对 6 只蟾蜍发出坐骨神经的脊骨迋节段腹角 运动神经元池的范围分别进行了统计, 结果见表 1 。 即以脊髓后正中沟为原点向腹侧 1174 1520 $\mu \mathrm{m}$, 向左（或右）侧 480 750 $\mu \mathrm{m}$ 的范围内均有较大的 运动神经元胞体 (图 1)。于是我们的微量注射点选 在运动神经元池的正中部, 即下针深度 $=(\mathrm{H} 1+\mathrm{H} 2)$ / 
$2 \approx 1350 \mu \mathrm{m}$, 旁开 (左侧) $=(\mathrm{L} 1+\mathrm{L} 2) / 2 \approx 620 \mu \mathrm{m}$ 。

\section{2 各组腓肠肌收缩形式的比较}

注射谷氨酸钠后，腓肠肌出现一段时间的持续 缩短现象, 肉眼观察腓肠肌呈不同程度的挛缩状 态, 即呈不同程度的强直收缩 (图 2)。其形态非常 类似于连续 $(50 \mathrm{~Hz})$ 电刺激离体坐骨神经腓肠肌标 本的坐骨神经时腓肠肌出现的强直收缩现象。收缩 曲线有一段时间的上升相，增加到最大后缓慢下 降; 从开始收缩到收缩幅度达峰值 (上升相) 约持 续 16 90 s, $1 \mathrm{~mol} / \mathrm{L}$ 组的上升相持续时间显著短于 其他组, 收缩最大张力也最大; 腓肠肌持续缩短时 间约为 $800 \sim 1500 \mathrm{~s}$, 组别之间差异不显著。最大 张力、张力变化速率与其他组相比具有显著差异。 $0.5 、 0.1 、 0.01 \mathrm{~mol} / \mathrm{L}$ 组之间现象差别不大, 腓肠肌 只出现一次程度较小的强直收缩现象。但上升相持 续时间、最大张力、张力变化速率、下降相持续时 间各不相同。生理盐水对照组均无腓肠肌缩短现 象。

\subsection{5 组腓肠肌收缩性的综合比较}

由表 2 可见, $1 \mathrm{~mol} / \mathrm{L}$ 组的上升相持续时间显著 短于其他各组 $(1 \mathrm{~mol} / \mathrm{L}$ 组分别与 $0.5 、 0.1 \mathrm{~mol} / \mathrm{L}$ 组 相比, $P<0.05)$, 与 $0.01 \mathrm{~mol} / \mathrm{L}$ 组差异极显著 $(P<0.01)$, 而 $0.5 、 0.1 、 0.01 \mathrm{~mol} / \mathrm{L}$ 组之间差异不显 著。最大张力: $1 \mathrm{~mol} / \mathrm{L}$ 组与 $0.5 、 0.1 、 0.01 \mathrm{~mol} / \mathrm{L}$ 组差异极显著; 0.5 和 $0.1 \mathrm{~mol} / \mathrm{L}$ 组之间差异不显著, 它们和 $0.01 \mathrm{~mol} / \mathrm{L}$ 组差异显著。张力变化速率: 1 $\mathrm{mol} / \mathrm{L}$ 组与其他各组差异极显著, 其他各组之间无 显著差异。下降相持续时间各组差异均不显著。

\section{3 讨 论}

哺乳动物中枢神经系统中, 谷氨酸广泛存在。 在神经冲动传递过程中, 突触前膜释放谷氨酸到突 触间隙, 与后膜上的谷氨酸受体结合, 进而引起突 触后神经元的兴奋。几毫秒内谷氨酸被邻近的神经 突起或胶质细胞吸收转化成谷氨酰胺, 终止了突触 后神经元的兴奋。后者又可分解出谷氨酸, 形成了 “谷氨酸一谷氨酰胺循环”(Levitan \& Kaczmarek, 2001)。

从本实验结果来看, 初步确定蟾蜍脊髓灰质腹 角运动神经元是谷氨酸敏感神经元。对照组腓肠肌 无收缩现象, 表明在本实验中腓肠肌的收缩是由于 蟾蜍脊髓灰质腹角的运动神经元上的谷氨酸受体 与谷氨酸结合, 引起了运动神经元的兴奋, 而不是
注射这种机械刺激引起的兴奋。引起腓肠肌收缩是 多个神经元共同兴奋的结果, 最大张力的大小由兴 奋的神经元数目决定，高浓度下更多的谷氨酸结合 了受体, 于是引发了较多的神经元兴奋, 表现出来 的腓肠肌张力、张力变化速率比较大。上升相持续 时间与谷氨酸钠和受体结合率有关, 高浓度组可以 尽快达到饱和，因此短于其他各组。各组下降相差

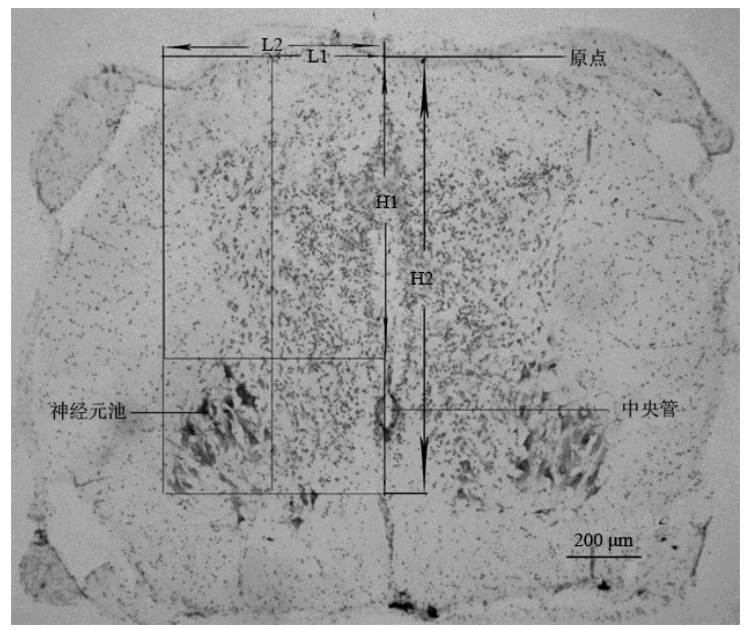

图 1 蜳蜍脊髓灰质腹角运动神经元池在切片上的位 置示意图

Fig. 1 The location of motor neuron pool in spinal cord gray matter coronal sections

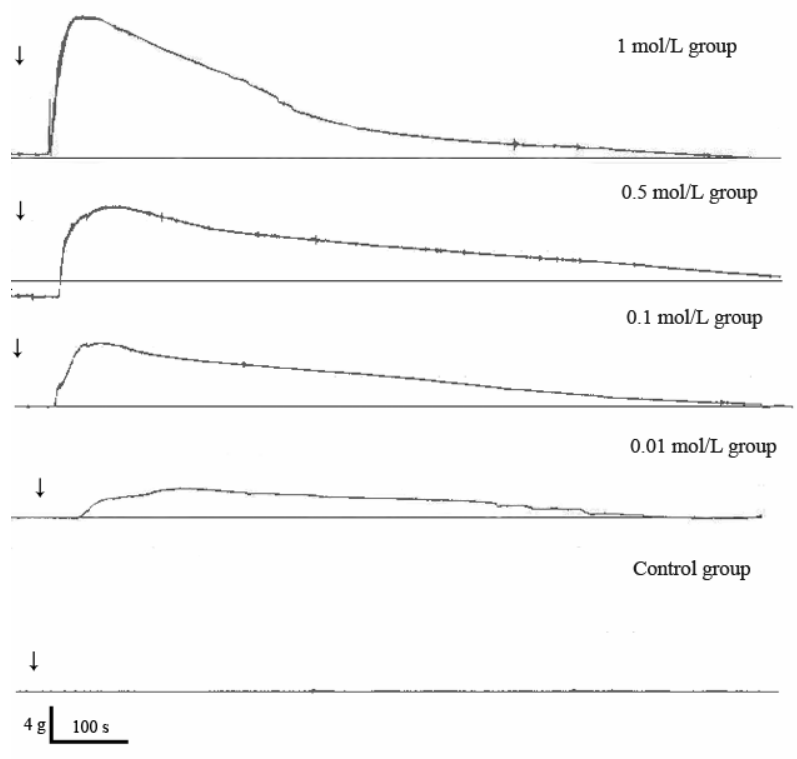

图 2 实验组和对照组腓肠肌收缩曲线

Fig. 2 Gastrocnemius contractility curve of experimental groups and control group

纵坐标: 收缩张力 $(\mathrm{g})$; 横坐标: 时间 ( $\mathrm{s})$ 。

Y-axis: Contractility tension (g); X-axis: Duration of contractility(s). 
表 1 蟾蜍腹角运动神经元池的定位区域

Tab. 1 The confines of motor neuron pool $(\mu \mathrm{m})$

\begin{tabular}{ccccccc}
\hline 编号 Number & L1 & L2 & H1 & H2 & L & H \\
\hline & 544 & 818 & 1081 & 1463 & 2611 & 1813 \\
2 & 535 & 746 & 1092 & 1546 & 2608 & 1934 \\
3 & 374 & 730 & 1211 & 1554 & 2292 & 1848 \\
4 & 530 & 763 & 1200 & 1561 & 2622 & 2008 \\
5 & 460 & 702 & 1291 & 1603 & 2328 & 1988 \\
6 & 435 & 737 & 1175 & 1377 & 2337 & 1812 \\
- & & & & & & \\
$\mathrm{x} \pm S$ & $480 \pm 67$ & $749 \pm 39$ & $1173 \pm 78$ & $1520 \pm 82$ & $2466 \pm 162$ & $1900 \pm 87$ \\
\hline
\end{tabular}

表 25 组腓肠肌收缩波各指标比较

Tab. 2 Comparison of gastrocnemius contractility curve in five groups

\begin{tabular}{lcccc}
\hline $\begin{array}{c}\text { 动物分组 Groups } \\
(\mathrm{mol} / \mathrm{L})(n=8)\end{array}$ & $\begin{array}{c}\text { 最大张力 }(\mathrm{g}) \\
\text { Maximal tension }\end{array}$ & $\begin{array}{c}\text { 上升相持续时间 }(\mathrm{s}) \\
\text { Duration of rising phase }\end{array}$ & $\begin{array}{r}\text { 张力变化速率 }(\mathrm{g} / \mathrm{s}) \\
\text { Velocity of tension variation }\end{array}$ & $\begin{array}{c}\text { 下降相持续时间 }(\mathrm{s}) \\
\text { Duration of descending phase }\end{array}$ \\
\hline Control & 0 & 0 & 0 & 0 \\
1 & $16.65 \pm 4.92$ & $36.5 \pm 17.7$ & $0.51 \pm 0.16$ & $1121 \pm 405$ \\
0.5 & $9.27 \pm 2.29^{* *}$ & $63.2 \pm 21.1^{*}$ & $0.16 \pm 0.09^{* *}$ & $1098 \pm 421$ \\
0.1 & $8.33 \pm 1.97^{* *}$ & $62.6 \pm 15.9^{*}$ & $0.03^{* *}$ & $1051 \pm 277$ \\
0.01 & $5.68 \pm 1.82^{* *+*}$ & $59.2 \pm 23.1^{* *}$ & $0.11 \pm 0.06^{* *}$ & $1239 \pm 356$ \\
\hline
\end{tabular}

与 $1 \mathrm{~mol} / \mathrm{L}$ 组比较, ${ }^{*} P<0.05,{ }^{* *} P<0.01$; 与 $0.5 \mathrm{~mol} / \mathrm{L}$ 组比较, ${ }^{+} P<0.05$; 与 $0.1 \mathrm{~mol} / \mathrm{L}$ 组比较, ${ }^{\#} P<0.05,{ }^{\# \#} P<0.01$ 。

Compare with $1 \mathrm{~mol} / \mathrm{L}$ group, ${ }^{*} P<0.05,{ }^{* *} P<0.01 ;$ compare with $0.5 \mathrm{~mol} / \mathrm{L}$ group, ${ }^{+} P<0.05$; compare with $0.1 \mathrm{~mol} / \mathrm{L}$ group, ${ }^{*} P<0.05$,

\#\# $P<0.01$.

别不显著是因为舒张时间和谷氨酸重吸收率有关。 另外, 本实验获得的腓肠肌收缩曲线与本实验室以 前研究相比, 连续电刺激 $(50 \mathrm{~Hz}, 20 \mathrm{~min})$ 蟾蜍坐 骨神经腓肠肌标本，坐骨神经对腓肠肌强直收缩的 波形比较相近, 但上升相持续时间长得多, 而与单 个电刺激坐骨神经引起的单收缩有很大的差别。这 提示谷氨酸一旦兴奋了运动神经元，运动神经元可 发放一定频率的冲动, 而不是单个冲动, 这与有机 磷农药中毒后神经肌肉接头间隙乙酰胆碱大量积 存，神经一肌肉接头后膜会持续兴奋的原理相似, 也表明谷氨酸钠要通过扩散, 逐渐与更多神经元结 合，而使得强直收缩的幅度逐渐增大。

高浓度的谷氨酸具有神经毒性, 主要是由于和 NMDA 受体结合后能引起急性细胞肿胀或激活各 种降解酶造成神经元溃变死亡, 这也是很多神经元 退变疾病的原因(Choi, 1994; Han, 1999)。对一般动
物的神经元来说, 引起兴奋的浓度一般在 $0.1 \mathrm{~mol} / \mathrm{L}$ 以下, 科学实验研究中一般使用浓度 $0.001 \sim 0.1$ $\mathrm{mol} / \mathrm{L}$ 来诱发神经核团的兴奋(Maureen et al, 2007; Cao et al, 2005)。对于不同的神经核团注射谷氨酸钠 的量不尽相同，一般在 0.02 0.1 $\mu \mathrm{L}$ 之间 (Lewis et al, 2002; Maureen et al, 2007)。在人脑突触间隙中， 1.1 $\mathrm{mmol} / \mathrm{L}$ 的谷氨酸足以使突触后膜上的谷氨酸受体 饱和, $1 \mathrm{~mol} / \mathrm{L}$ 已属于高浓度, 会损伤神经元, 但 是在本实验中， $1 \mathrm{~mol} / \mathrm{L}$ 却引起腓肠肌的收缩幅度 最高, 证明与其他动物相比, 蟾蜍脊髓灰质腹角对 谷氨酸钠具有很高的耐受性。

另外，本文提供了蟾蜍脊髓腰骮膨大处的左右 径、背腹径的统计数据以及灰质腹角运动神经元池 的精确定位坐标，对今后以蟾蜍为实验材料进行神 经生物学、比较解剖生理学的研究均具有很好的参 考价值。 


\section{参考文献：}

Ai HB. 2004. Experimental Course of Human Anatomy and Physiology[M]. Beijing:Science Publishing House, 162-164. 艾洪滨. 2004. 人体解剖 生理学实验教程. 北京: 科学出版社, 162-164.

Cai L, Cui XY, Ai HB. 2007. Research of neurone architecture of spinal gray matter and spinal nerve in Bufo bufogargarizans[J]. J Shandong Normal Univ, 22(3): 104-106. [ 蔡 雷, 崔希云, 艾洪滨. 2007. 中华 大蟾蜍脊䯕灰质神经元构筑和脊神经的研究. 山东师范大学学报: 自然科学版, 22(3): 104-106.

Cao DY, Guo Y, Zhang Q, Tian YL, Wang HS, Zhao Y. 2005. Effects of glutamate on the afferent discharges of dorsal cutaneous sensory nerves in rats[J]. Neurosci Bull, 21(2): 111-115.

Choi DW. 1994. Glutamate receptors and the induction of excitotoxicity neuronal death[J]. Prog Brain Res, 100: 47-51.

Han JS. 1999. Principles of Neural Science[M].Beijing: Beijing Medical University Publishing House, 524-537. [韩济生. 1999. 神经科学原理. 北京: 北京医科大学出版社, 524-537.]

Levitan LB, Kaczmarek LK. 2001. The Neuron: Cell and Molecular Biology[M]. Su SY, Bao XM, Trans. Beijing: Science Publishing House, 154-155 [莱维坦 L B, 卡茨玛克 LK. 2001. 神经元: 细胞和分 子生物学 $[\mathrm{M}]$, 舒斯云, 包新民, 译. 北京: 科学出版社, 154-155.

Lewis MW, Hermann GE, Rogers RC, Travagli RA. 2002. In vitro and in vivo analysis of the effects of corticotropin releasing factor on rat dorsal vagal complex[J]. J Physiol, 543: 135-146.

Maureen TC, Erin CM, Niaz S, Joseph G.V, Richard AG. 2007. A reevaluation of the effects of stimulation of the dorsal motor nucleus of the vagus on gastric motility in the rat[J]. Am J Physiol Regul Integr Comp Physiol, 292: R291-R307.

Spencer SE, Talman WT. 1986. Central modulation of gastric pressure by substance P: a comparison with glutamate and acetylcholine[J]. Brain Res, 385: 371-374.

Usherwood PN, Machili P. 1969. Insect glutamate receptors [J]. Nature, 210: 634-636.

Wan XC, Yang TZ, Xu CT. 1999. Modern Neurobiology[M]. Beijing: Beijing Medical University: Peking Union Medical College Publishing House, 160-162.[万选才, 杨天祝, 徐承奉. 1999. 现代神经生物学. 北京: 北京医科大学、中国协和医科大学联合出版社, 160-162.

Wang LX, Martinezl V, Laranche M, Tache Y. 2009. Proximal colon distension induces Fos expression in oxytocin-,vasopressin-, CRF- and catecholamines-containing neurons in rat brain[J]. Brain Res, 247: 79-92.

Wang WG, Chen X, Jiang H, Jiang ZY. 2008. Effects of ghrelin on glucose-sensing and gastric distension sensitive neurons in rat dorsal vagal complex[J]. Regulatory Pept, 146: 169-175.

Zeng P, Sun YG, Ai HB. 2007. Comparative research of the physiological characteristics of isolated small intestine sections from some kinds of vertebrates[J]. J Shandong Normal Univ, 22(1): 119-121. [曾 鹏, 孙 一, 艾洪滨. 2007. 鲫鱼、蟾蜍、鳖、家鸽离体小肠段生理特性的比 较. 山东师范大学学报: 自然科学版 22(1): 119-121.]

\section{山东师范大学生命科学学院动物生理研究室简介}

本研究室主要运用免疫细胞化学技术、电生理技术、大鼠脑立体定位技术、脑机接口等技术进行应激 状态下胃肠机能的中枢调控机制（参与调控的中枢核团、神经元放电、神经通路、神经递质及受体等）、 清醒大鼠执行某种行为时大脑皮层感觉运动区神经元电信号编码、心脏电生理以及脊椎动物比较生理等方 面的研究。已完成项目有疑核对胃运动和胃酸分泌调控作用及其机制的研究（山东省自然科学基金, Y2002D18)、高频心电信息中连续高频段形成机理的研究（山东省自然科学基金，Y97D18077）、心电时域 频域分析用于诊断病毒性心肌炎的研究 (山东省科委项目, 93228201)、脑机接口基础研究一一神经信号 的采集与处理（山东省自然科学基金项目, Y2007C029)。目前正在进行的研究课题有 “大鼠迷走复合体在 束缚一浸水应激致胃粘膜损伤及胃运动光进中的作用及其机制的研究”（国家自然科学基金项目， 30770277)，“大鼠下丘脑视上核在束缚-浸水应激致胃机能紊乱中的作用及其机制的研究” (国家自然科学 基金项目，30970354）。已在国际 SCI、EI 刊物、国内生理学专业高级刊物《生理学报》、《中国生物医学 工程学报》(英文版)、《中国应用生理学杂志》、《生理科学进展》、《神经解剖学杂志》、《动物学研究》、《动 物学杂志》等刊物发表研究论文 100 余篇。 